\title{
No existencia global para un sistema de ecuaciones de onda viscoelástica no lineal con operador p-Laplaciano
}

\author{
Teófanes Quispe Méndez ${ }^{1}$
}

Resumen: Consideramos un problema mixto para un sistema de ecuaciones de onda viscoelástica no lineal con operador p-Laplaciano. Bajo ciertas condiciones sobre las funciones de relajación y los términos de fuente, utilizando el método indirecto, probamos un resultado de no existencia global para ciertas soluciones con energía inicial no positiva así como con energía inicial positivos.

Palabras claves: Sistema de ecuaciones de onda viscoelástica no lineal; operador p-Laplaciano; no existencia global; explosión de soluciones.

\section{Global Nonexistence for a system of nonlinear viscoelastic wave equations with p-Laplacian operator}

\begin{abstract}
We consider a mixed problem for a system of nonlinear viscoelastic wave equations with p-Laplacian operator. Under certain conditions on the relaxation functions and the source terms, using the indirect method, we prove a result of global nonexistence for certain solutions with nonpositive initial energy as well as with positive initial energy.
\end{abstract}

Keywords: System of nonlinear viscoelastic wave equations; p-Laplacian operator; global nonexistence; blow-up of solutions.

Recibido: 08/09/2018. Aceptado: 18/11/2018. Publicado online: 31/12/2018.

(C)Los autores. Este artículo es publicado por la Revista PESQUIMAT de la Facultad de Ciencias Matemáticas, Universidad Nacional Mayor de San Marcos. Este es un artículo de acceso abierto, distribuido bajo los términos de la licencia Creative Commons Atribucion-No Comercia-Compartir Igual 4.0 Internacional.(http://creativecommons.org/licenses/by-nc-sa/4.0/) que permite el uso no comercial, distribución y reproducción en cualquier medio, siempre que la obra original sea debidamente citada. Para información, por favor póngase en contacto con revistapesquimat.matematica@unmsm.edu.pe

\footnotetext{
${ }^{1}$ UNMSM, Facultad de Ciencias Matemáticas. e-mail: tquispem@unmsm.edu.pe.
} 


\section{Introducción}

En este artículo, consideramos el problema para el siguiente sistema de ecuaciones de onda viscoelástica no lineal con operador p-Laplaciano:

$$
\begin{gathered}
\left.\left|u^{\prime}\right|^{\rho} u^{\prime \prime}-\Delta u-\Delta_{p} u-\Delta u^{\prime \prime}+g_{1} * \Delta u-\Delta u^{\prime}=f_{1}(u, v) \text { en } \Omega \times\right] 0, \infty[, \\
\left.\left|v^{\prime}\right|^{\rho} v^{\prime \prime}-\Delta v-\Delta_{p} v-\Delta v^{\prime \prime}+g_{2} * \Delta v-\Delta v^{\prime}=f_{2}(u, v) \text { en } \Omega \times\right] 0, \infty[,
\end{gathered}
$$

con condiciones iniciales

$$
\begin{aligned}
& u(x, 0)=u_{0}(x), u^{\prime}(x, 0)=u_{1}(x) \text { en } \Omega, \\
& v(x, 0)=v_{0}(x), v^{\prime}(x, 0)=v_{1}(x) \text { en } \Omega,
\end{aligned}
$$

y condiciones de frontera

$$
\begin{aligned}
& u(x, t)=0 \text { en } \partial \Omega \times] 0, \infty[, \\
& v(x, t)=0 \text { en } \partial \Omega \times] 0, \infty[,
\end{aligned}
$$

donde $\Omega$ es un conjunto abierto y acotado de $\mathbb{R}^{n}(n \geq 1)$ con frontera regular $\partial \Omega, \Delta$ es el operador Laplaciano, $\Delta_{p}$ es el operador p-Laplaciano definido por

$$
\Delta_{p} w=\operatorname{div}\left(|\nabla w|^{p-2} \nabla w\right)
$$

con $p \geq 2, \nabla$ es el operador gradiente, div es el operador divergencia, $\rho$ es un número real positivo que cumple cierta condición. Las funciones $u_{0}, u_{1}, v_{0}$ y $v_{1}$ son datos iniciales dados. Las funciones $g_{1}(t)$ y $g_{2}(t)$ son reales positivas diferenciables para $t \geq 0$ y las funciones $f_{1}(s, r) \mathrm{y}$ $f_{2}(s, r)$ son reales no lineales para $(s, r) \in \mathbb{R}^{2}$ y el operador $*$ definido por

$$
(g * w)(x, t)=\int_{0}^{t} g(t-s) w(x, s) d s .
$$

Las derivadas parciales con respecto al tiempo denotamos: $u^{\prime}=\frac{\partial u}{\partial t} \mathrm{y} u^{\prime \prime}=\frac{\partial^{2} u}{\partial t^{2}}$.

Este problema tiene su origen en la descripción matemática de los materiales viscoelásticos y describe la interacción de dos campos escalares. Es bien sabido que los materiales viscoelásticos exhiben amortiguación natural, que es debido a la propiedad especial de estos materiales para retener una memoria de su historia pasada. Desde el punto de vista matemático, estos efectos de amortiguación se modelan por los operadores integro-diferenciales. Por tanto, la dinámica de los materiales viscoelásticos son de gran importancia e interés ya que tienen amplias aplicaciones en la física y la ingeniería. Más información al respecto, ver [3, 5, 16].

Nuestro trabajo esta motivado por el sistema propuesto por Segal [17]

$$
\left\{\begin{array}{l}
u^{\prime \prime}-\Delta u+m_{1}^{2} u+k_{1}^{2} u v^{2}=0 \\
v^{\prime \prime}-\Delta v+m_{2}^{2} v+k_{2}^{2} u^{2} v=0
\end{array}\right.
$$

que surge en el estudio de la teoría del campo cuántico y describe la interacción de los mesones $u$ y $v$, donde $m_{1}$ y $m_{2}$ son las masas de estos mesones respectivamente, $k_{1}$ y $k_{2}$ son las constantes de interacción. El siguiente sistema de ecuaciones de onda con término fuente no lineal

$$
\left\{\begin{array}{l}
u^{\prime \prime}-\Delta u+\alpha_{1} u+\beta_{1}|v|^{p+2}|u|^{p} u=f_{1}, \\
v^{\prime \prime}-\Delta v+\alpha_{2} v+\beta_{2}|u|^{p+2}|v|^{p} v=f_{2}
\end{array}\right.
$$


fue estudiado por Medeiros y Miranda [12, 13]. Se tiene también el siguiente sistema de ecuaciones de onda viscoelástica con términos disipativo y fuente

$$
\left\{\begin{array}{l}
u^{\prime \prime}-\Delta u+\int_{0}^{t} g_{1}(t-s) \Delta u(s) d s-\Delta u^{\prime}=f_{1}(u, v), \\
v^{\prime \prime}-\Delta v+\int_{0}^{t} g_{1}(t-s) \Delta v(s) d s-\Delta v^{\prime}=f_{2}(u, v)
\end{array}\right.
$$

que fue investigado por Liang y Gao [7] y demostraron la existencia global, el decaimiento exponencial y la explosión de soluciones; también Andrade y Mognon [2], sin términos disipativo, establecieron la existencia y la unicidad global; Sun y Wang [18], sin términos de viscoelasticidad, demostraron la existencia local y global, el decaimiento exponencial y la explosión de soluciones en tiempo finito. Posteriormente se tiene el siguiente sistema

$$
\left\{\begin{array}{l}
\left|u^{\prime}\right|^{\rho} u^{\prime \prime}-\Delta u-\Delta u^{\prime \prime}-\Delta u^{\prime}=f_{1}(u, v) \\
\left|v^{\prime}\right|^{\rho} v^{\prime \prime}-\Delta v-\Delta v^{\prime \prime}-\Delta v^{\prime}=f_{2}(u, v)
\end{array}\right.
$$

el cual fue estudiado por Liu [10] y estableció la existencia global y el decaimiento exponencial. El siguiente sistema

$$
\left\{\begin{array}{l}
\left|u^{\prime}\right|^{\rho} u^{\prime \prime}-\Delta u-\Delta u^{\prime \prime}+\int_{0}^{t} g_{1}(t-s) \Delta u(s) d s-\Delta u^{\prime}=f_{1}(u, v), \\
\left|v^{\prime}\right|^{\rho} v^{\prime \prime}-\Delta v-\Delta v^{\prime \prime}+\int_{0}^{t} g_{1}(t-s) \Delta v(s) d s-\Delta v^{\prime}=f_{2}(u, v),
\end{array}\right.
$$

fue estudiado por Quispe et al. [15] y ellos probaron la existencia local y la no existencia global con energía inicial no positiva y como también con energía inicial positiva. También el sistema (1.11) fue investigado por Liu y Yu [11], quienes establecieron la existencia local y global, y el decaimiento exponencial.

El problema (1.1) - (1.6) con términos disipativos no lineales, Hao et al. [6] probaron la no existencia global con energía inicial negativa.

Motivados por los trabajos anteriores, estudiamos el problema (1.1) - (1.6) y mostramos la no existencia global de soluciones, mediante el método indirecto con energía inicial acotada por cierta constante positiva. En la discusión del problema (1.1) - (1.6), emplearemos las estrategias y técnicas inspiradas en los trabajos de Liu [9] y Quispe et al. [15].

El trabajo esta organizado de la siguiente manera. En la Sección 2, se explican algunos conceptos y notaciones a utilizarse, se dan resultados especiales sin demostración y se imponen las hipótesis técnicas para el desarrollo del trabajo. En la sección 3, enunciamos algunos lemas importantes y el teorema principal del trabajo con sus correspondientes demostraciones.

\section{Preliminares}

En esta sección presentamos algunos conceptos (mayor información, ver Lions [8]), notaciones, hipótesis y resultados sin demostración, los cuales serán usados en el desarrollo del presente trabajo.

Sea $\Omega$ un conjunto abierto y acotado de $\mathbb{R}^{n}$ con frontera regular $\partial \Omega$. Denotamos el producto interno en $L^{2}(\Omega)$ por

$$
(u, v)=\int_{\Omega} u(x) v(x) d x
$$


Denotamos por $|\cdot|_{p}$ la norma usual en el espacio $L^{p}(\Omega)$ para $1 \leq p \leq \infty$. Consideramos el espacio de Hilbert $H_{0}^{1}(\Omega)$, provisto con el producto interno y norma dado por

$$
((u, v))=\int_{\Omega} \nabla u(x) \cdot \nabla v(x) d x \quad \mathrm{y} \quad\|u\|=\left(\int_{\Omega}|\nabla u(x)|^{2} d x\right)^{\frac{1}{2}} .
$$

En el espacio de Sobolev $W_{0}^{1, p}(\Omega)$ usamos la norma

$$
\|u\|_{1, p}=\left(\int_{\Omega}|\nabla u(x)|^{p} d x\right)^{\frac{1}{p}} .
$$

Sea $X$ un espacio de Banach, $T$ y $p$ números reales tales que $0<T \leq \infty$ y $1 \leq p \leq \infty$. Representamos con $L^{p}(0, T ; X)$ al espacio de Banach de las funciones vectoriales $\left.u:\right] 0, T[\longrightarrow$ $X$ medibles con $\|u(t)\|_{X} \in L^{p}(0, T)$, provisto de la norma

$$
\begin{gathered}
\|u\|_{L^{p}(0, T ; X)}=\left(\int_{0}^{T}\|u(t)\|_{X}^{p} d t\right)^{\frac{1}{p}}, 1 \leq p<\infty, \\
\|u\|_{L^{\infty}(0, T ; X)}=\sup _{0<t<T} \operatorname{ess}\|u(t)\|_{X}, p=\infty .
\end{gathered}
$$

Similarmente, cuando $0<T<\infty$, representamos con $C([0, T] ; X)$ al espacio de Banach de las funciones continuas $u:[0, T] \longrightarrow X$, provisto de la norma

$$
\|u\|_{C([0, T] ; X)}=\sup _{0 \leq t \leq T}\|u(t)\|_{X} .
$$

Denotamos las derivadas parciales con respecto al tiempo $w^{\prime}=\frac{\partial w}{\partial t}$ y $w^{\prime \prime}=\frac{\partial^{2} w}{\partial t^{2}}$, y escribimos $w(t)(x)=w(x, t)$.

Hipótesis. Imponemos sobre el número $\rho$ y las funciones reales $g_{1}, g_{2}, f_{1}$ y $f_{2}$ las siguientes condiciones:

$\left(H_{1}\right) \rho$ es un número real tal que

$$
0<\rho \leq \frac{2}{n-2} \text { si } n \geq 3 \text { y } \rho>0 \text { si } n=1,2 .
$$

$\left(H_{2}\right) g_{1}, g_{2}:[0, \infty) \rightarrow[0, \infty)$ son funciones no crecientes acotadas de clase $\mathcal{C}^{1}$ tales que

$$
\begin{aligned}
& g_{1}(0)>0, \quad 1-\int_{0}^{\infty} g_{1}(s) d s=l_{1}>0, \\
& g_{2}(0)>0, \quad 1-\int_{0}^{\infty} g_{2}(s) d s=l_{2}>0 .
\end{aligned}
$$

$\left(H_{3}\right) f_{1}, f_{2}: \mathbb{R}^{2} \rightarrow \mathbb{R}$ son funciones continuas y existe una constante $d>0$ tal que

$$
\begin{aligned}
& \left|f_{1}(u, v)\right| \leq d\left(|u+v|^{r-1}+|u|^{\frac{r}{2}-1}|v|^{\frac{r}{2}}\right), \forall(u, v) \in \mathbb{R}^{2}, \\
& \left|f_{2}(u, v)\right| \leq d\left(|u+v|^{r-1}+|u|^{\frac{r}{2}}|v|^{\frac{r}{2}-1}\right), \forall(u, v) \in \mathbb{R}^{2},
\end{aligned}
$$

donde

$$
r>p \geq 2 \text { si } n=1,2 \text { y } 2 \leq p<r \leq \frac{2(n-1)}{n-2} \text { si } n \geq 3 .
$$


$\left(H_{4}\right)$ Existe una función $F: \mathbb{R}^{2} \rightarrow[0, \infty)$ diferenciable tal que

$$
\frac{\partial F}{\partial u}=f_{1}(u, v) \text { y } \frac{\partial F}{\partial v}=f_{2}(u, v), \forall(u, v) \in \mathbb{R}^{2}
$$

existen constantes $c_{0}, c_{1}>0$ tales que

$$
c_{0}\left[|u|^{r}+|v|^{r}\right] \leq u f_{1}(u, v)+v f_{2}(u, v)=r F(u, v) \leq c_{1}\left[|u|^{r}+|v|^{r}\right], \forall(u, v) \in \mathbb{R}^{2} ;
$$

y para cada $\lambda \geq 0$ se tiene

$$
F(\lambda u, \lambda v)=\lambda^{r} F(u, v), \forall(u, v) \in \mathbb{R}^{2},
$$

donde $r$ verifica (2.1).

Observación 2.1. Las funciones que satisface $\left(H_{3}\right)$ y $\left(H_{4}\right)$, puede ser tomado como por ejemplo $(\operatorname{ver}[1])$

$$
F(u, v)=\frac{1}{r}\left[a|u+v|^{r}+2 b|u v|^{\frac{r}{2}}\right]
$$

donde $a, b>0$ son constantes reales, así

$$
\begin{aligned}
& f_{1}(u, v)=a|u+v|^{r-2}(u+v)+b|u|^{\frac{r}{2}-2}|v|^{\frac{r}{2}} u, \\
& f_{2}(u, v)=a|u+v|^{r-2}(u+v)+b|v|^{\frac{r}{2}-2}|u|^{\frac{r}{2}} v .
\end{aligned}
$$

Lema 2.2 (Desigualdad de Sobolev-Poincaré [4]). Si $1 \leq q \leq \frac{n p}{n-p}$ para $1 \leq p<n$ y $1 \leq q<\infty$ para $n \leq p$, entonces existe una constante $C_{*}>0$ tal que

$$
|u|_{q} \leq C_{*}\|u\|_{1, p}, \forall u \in W_{0}^{1, p}(\Omega) .
$$

Lema 2.3 (Desigualdad generalizada de Gronwall [14]). Sea $f:[0, \infty) \rightarrow[0, \infty)$ continua, $g:] 0, \infty[\rightarrow] 0, \infty[$ continua y no decreciente y sea $C$ una constante positiva. Si

$$
f(t) \leq C+\int_{0}^{t} g(f(s)) d s, 0 \leq t<\infty,
$$

entonces

$$
f(t) \leq G^{-1}\left(T_{*}\right)<\infty, 0 \leq t \leq T_{*},
$$

para cualquier número fijo $T_{*}<G(\infty)$, donde

$$
G(\tau)=\int_{C}^{\tau} \frac{1}{g(s)} d s, \text { para } \tau \geq C .
$$

Además, si $G(\infty)=\infty$, entonces

$$
f(t) \leq G^{-1}(t), \text { para todo } t \geq 0 .
$$

Diferenciando el operador $g \square w$ y haciendo algunos cálculos, se obtiene el siguiente resultado.

Lema 2.4. Si $g \in C^{1}\left(\left[0, \infty[) y w \in C^{1}\left([0, T] ; L^{2}(\Omega)\right)\right.\right.$, entonces

$$
\begin{aligned}
\int_{0}^{t} g(t-s)\left(w(s), w^{\prime}(t)\right) d s= & -\frac{1}{2} \frac{d}{d t}\left[(g \square w)(t)-\left(\int_{0}^{t} g(s) d s\right)|w(t)|_{2}^{2}\right] \\
& -\frac{1}{2} g(t)|w(t)|_{2}^{2}+\frac{1}{2}\left(g^{\prime} \square w\right)(t),
\end{aligned}
$$

donde

$$
(h \square w)(t):=\int_{0}^{t} h(t-s)|w(t)-w(s)|_{2}^{2} d s .
$$




\section{No Existencia Global}

En esta sección, estudiamos la propiedad de explosión de soluciones en tiempo finito del problema (1.1) - (1.6). En la discusión emplearemos el método indirecto como Liu [9] y Quispe et al. [15].

La energía del problema (1.1) - (1.6) se define por

$$
\begin{aligned}
E(t)= & \frac{1}{\rho+2}\left(\left|u^{\prime}(t)\right|_{\rho+2}^{\rho+2}+\left|v^{\prime}(t)\right|_{\rho+2}^{\rho+2}\right)+\frac{1}{2}\left(\left\|u^{\prime}(t)\right\|^{2}+\left\|v^{\prime}(t)\right\|^{2}\right) \\
& +\frac{1}{p}\left(\|u(t)\|_{1, p}^{p}+\|v(t)\|_{1, p}^{p}\right)+\frac{1}{2}\left(G_{1}(t)\|u(t)\|^{2}+G_{2}(t)\|v(t)\|^{2}\right) \\
& +\frac{1}{2}\left(\left(g_{1} \square \nabla u\right)(t)+\left(g_{2} \square \nabla v\right)(t)\right)-\int_{\Omega} F(u(t), v(t)) d x,
\end{aligned}
$$

para $t \geq 0$, donde

$$
G_{i}(t)=1-\int_{0}^{t} g_{i}(\tau) d \tau, i=1,2
$$

Lema 3.1. Supongamos que $\left(H_{1}\right)-\left(H_{4}\right)$ se verifican, $u_{0}, v_{0} \in W_{0}^{1, p}(\Omega)$ y $u_{1}, v_{1} \in H_{0}^{1}(\Omega)$. Si $(u, v)$ es una solución del problema $(1.1)-(1.6)$, entonces la energía $E(t)$ es una función no creciente para $t \geq 0$,

$$
\begin{aligned}
E^{\prime}(t)= & -\left(\left\|u^{\prime}(t)\right\|^{2}+\left\|v^{\prime}(t)\right\|^{2}\right) \\
& -\frac{1}{2}\left(g_{1}(t)|\nabla u(t)|_{2}^{2}+g_{2}(t)|\nabla v(t)|_{2}^{2}\right)+\frac{1}{2}\left(\left(g_{1}^{\prime} \square \nabla u\right)+\left(g_{2}^{\prime} \square \nabla u\right)\right) \leq 0
\end{aligned}
$$

$y$

$$
E(t)+\int_{0}^{t}\left(\left\|u^{\prime}(s)\right\|^{2}+\left\|v^{\prime}(s)\right\|^{2}\right) d s \leq E(0)
$$

donde

$$
\begin{aligned}
E(0)= & \frac{1}{\rho+2}\left(\left|u_{1}\right|_{\rho+2}^{\rho+2}+\left|v_{1}\right|_{\rho+2}^{\rho+2}\right)+\frac{1}{2}\left(\left\|u_{1}\right\|^{2}+\left\|v_{1}\right\|^{2}\right) \\
& +\frac{1}{p}\left(\left\|u_{0}\right\|_{1, p}^{p}+\left\|v_{0}\right\|_{1, p}^{p}\right) \\
& +\frac{1}{2}\left(\left\|u_{0}\right\|^{2}+\left\|v_{0}\right\|^{2}\right)-\int_{\Omega} F\left(u_{0}(x), v_{0}(x)\right) d x .
\end{aligned}
$$

Demostración. Multiplicando (1.1) por $u^{\prime}$ y (1.2) por $v^{\prime}$, integrando sobre $\Omega$ y utilizando (1.3) - (1.6) con el teorema de la divergencia, se obtiene (3.2). La desigualdad (3.3) es una consecuencia directa de (3.2).

Lema 3.2. Supongamos que $\left(H_{4}\right)$ se verifica. Entonces existe $\eta>0$ tal que para cada $(u, v) \in$ $H_{0}^{1}(\Omega) \times H_{0}^{1}(\Omega)$ se tiene

$$
\int_{\Omega} F(u, v) d x \leq \eta\left(l_{1}\|u(t)\|^{2}+l_{2}\|v(t)\|^{2}\right)^{\frac{r}{2}}
$$

donde $l_{1}$ y $l_{2}$ son las constantes dadas en $\left(H_{2}\right)$. 
Demostración. Usando la desigualdad de Sobolev-Poincaré, tenemos

$$
\begin{aligned}
\int_{\Omega} F(u, v) d x & \leq \frac{c_{1}}{r}\left(|u|_{r}^{r}+|v|_{r}^{r}\right) \\
& \leq \frac{c_{1}}{r} C_{*}^{r}\left(\|u\|^{r}+\|v\|^{r}\right) \\
& \leq \frac{c_{1}}{r} C_{*}^{r}\left(\|u\|^{2}+\|v\|^{2}\right)^{\frac{r}{2}} \\
& \leq \frac{c_{1}}{r} C_{*}^{r}\left(\operatorname{máx}\left\{\frac{1}{l_{1}}, \frac{1}{l_{2}}\right\}\right)^{\frac{r}{2}}\left(l_{1}\|u\|^{2}+l_{2}\|v\|^{2}\right)^{\frac{r}{2}}
\end{aligned}
$$

Tomando $\eta=\frac{c_{1}}{r} C_{*}^{r}\left(\operatorname{máx}\left\{\frac{1}{l_{1}}, \frac{1}{l_{2}}\right\}\right)^{\frac{r}{2}}$, completa la prueba.

Definamos los siguientes funcionales

$$
\begin{gathered}
\Gamma(t)=\Gamma(u(t), v(t))=G_{1}(t)\|u(t)\|^{2}+G_{2}(t)\|v(t)\|^{2}+\left(g_{1} \square \nabla u\right)(t)+\left(g_{2} \square \nabla v\right)(t), \\
I(t)=I(u(t), v(t))=\Gamma(t)-r \int_{\Omega} F(u(t), v(t)) d x, \\
J(t)=J(u(t), v(t))=\frac{1}{2} \Gamma(u(t), v(t))-\int_{\Omega} F(u(t), v(t)) d x .
\end{gathered}
$$

Entonces

$$
\begin{aligned}
E(t)= & \frac{1}{\rho+2}\left(\left|u^{\prime}(t)\right|_{\rho+2}^{\rho+2}+\left|v^{\prime}(t)\right|_{\rho+2}^{\rho+2}\right)+\frac{1}{2}\left(\left\|u^{\prime}(t)\right\|^{2}+\left\|v^{\prime}(t)\right\|^{2}\right) \\
& +\frac{1}{p}\left(\|u(t)\|_{1, p}^{p}+\|v(t)\|_{1, p}^{p}\right)+J(u(t), v(t)) .
\end{aligned}
$$

Para $t \geq 0$, definamos

$$
d_{1}(t)=\inf _{\substack{(u, v) \in H_{0}^{1}(\Omega) \times H_{0}^{1}(\Omega) \\(u, v) \neq(0,0)}} d_{2}(u, v)
$$

donde

$$
d_{2}(u, v)=\sup _{\lambda \geq 0} J(\lambda u, \lambda v)
$$

Lema 3.3. Supongamos que $\left(H_{4}\right)$ se verifica. Entonces para $t \geq 0$ se tiene

$$
0<d \leq d_{1}(t) \leq d_{2}(u, v)
$$

$y$

$$
d_{2}(u, v)=\frac{r-2}{2 r}\left[\frac{(\Gamma(t))^{\frac{r}{r-2}}}{\left(r \int_{\Omega} F(u, v) d x\right)^{\frac{2}{r-2}}}\right]
$$

donde

$$
d=\frac{r-2}{2 r}\left(\frac{1}{\eta r}\right)^{\frac{2}{r-2}}
$$

y $\eta$ es la constante del Lema 3.2. 
Demostración. Para $(u, v) \in H_{0}^{1}(\Omega) \times H_{0}^{1}(\Omega)$ fijo, $(u, v) \neq(0,0)$, definamos la función

$$
\varphi(\lambda)=J(\lambda u, \lambda v)=\frac{\lambda^{2}}{2} \Gamma(u, v)-\lambda^{r} \int_{\Omega} F(u, v) d x .
$$

Entonces

$$
\varphi^{\prime}\left(\lambda_{0}\right)=0, \quad \text { para } \lambda_{0}=\left(\frac{\Gamma(t)}{r \int_{\Omega} F(u, v) d x}\right)^{\frac{1}{r-2}},
$$

donde $\int_{\Omega} F(u, v) d x>0$ para $(u, v) \neq(0,0)$, por $\left(H_{4}\right)$. Por consiguiente

$$
\sup _{\lambda \geq 0} \varphi(\lambda)=\varphi\left(\lambda_{0}\right)=d_{2}(u, v) .
$$

Entonces de (3.6) el resultado se obtiene usando la desigualdad (3.4) y desde que $G_{i}>l_{i}, i=1,2$.

Lema 3.4. Supongamos que $\left(H_{1}\right)-\left(H_{4}\right)$ se verifican. Si para cada número fijo $\delta<1$, asumamos que $u_{0}, v_{0} \in W_{0}^{1, p}(\Omega), u_{1}, v_{1} \in H_{0}^{1}(\Omega)$ y satisface

$$
E(0)<\delta d \text { y } I(0)<0,
$$

donde d está dado por (3.5). Entonces

$$
I(t)<0, \text { para cada } t>0
$$

$y$

$$
d<\frac{r-2}{2 r} \Gamma(t)<\frac{r-2}{2} \int_{\Omega} F(u, v) d x, \text { para cada } t>0 .
$$

Demostración. Por (3.3) y (3.7), resulta

$$
E(t)<\delta d, \text { para cada } t>0 .
$$

Ahora veamos que se cumple $I(t)<0$, para cada $t>0$. Razonando por el absurdo, supongamos que existe un número $t_{1}>0$ tal que $I\left(t_{1}\right) \geq 0$. Desde que $u(t)$ y $v(t)$ son continuas, se tiene que $I(t)$ es continua. Por lo cual, existe $\left.\left.t_{*} \in\right] 0, t_{1}\right]$ tal que $I\left(t_{*}\right)=0$ y $I(t)<0$, para $0 \leq t<t_{*}$. Por consiguiente

$$
\Gamma(t)<r \int_{\Omega} F(u(t), v(t)) d x, \text { para } 0 \leq t<t_{*} .
$$

Entonces, usando el Lema 3.3, se obtiene

$$
d<\frac{r-2}{2 r} \Gamma(t), \text { para } 0 \leq t<t_{*} .
$$

Del cual

$$
d<\frac{r-2}{2} \int_{\Omega} F(u(t), v(t)) d x, \text { para } 0 \leq t<t_{*} .
$$

Desde que $t \longmapsto \int_{\Omega} F(u(t), v(t)) d x$ es continua, tenemos $\int_{\Omega} F\left(u\left(t_{*}\right), v\left(t_{*}\right)\right) d x \neq 0$. En vista del Lema 3.3 y $I\left(t_{*}\right)=0$, resulta

$$
d \leq \frac{r-2}{2} \int_{\Omega} F\left(u\left(t_{*}\right), v\left(t_{*}\right)\right) d x=\frac{r-2}{2 r} \Gamma\left(t_{*}\right)=J\left(u\left(t_{*}\right), v\left(t_{*}\right)\right),
$$

el cual es imposible, desde que $J\left(u\left(t_{*}\right), v\left(t_{*}\right)\right) \leq E\left(t_{*}\right) \leq \delta d<d$. Por tanto se verifica (3.8). La desigualdad (3.9) se obtiene usando (3.8) y Lema 3.3. Esto completa la demostración. 
Lema 3.5. Supongamos que (2.1) se verifica. Entonces existe una constante $C>0$ tal que

$$
|w|_{r}^{q} \leq C\left[\|w\|^{2}+|w|_{r}^{r}\right]
$$

para todo $w \in H_{0}^{1}(\Omega)$ y $2 \leq q \leq r$.

Demostración. Si $|w|_{r} \leq 1$, y usando la desigualdad de Sobolev-Poincaré, resulta

$$
|w|_{r}^{q} \leq|w|_{r}^{2} \leq C_{*}\|w\|^{2} \leq C_{*}\left[\|w\|^{2}+|w|_{r}^{r}\right] .
$$

Si $|w|_{r}>1$, entonces

$$
|w|_{r}^{q} \leq|w|_{r}^{r} \leq|w|_{r}^{r}+\|w\|^{2}
$$

Así se tiene (3.10).

Consideremos

donde $\hat{\delta}=\operatorname{máx}\{0, \delta\}$. Entonces

$$
H(t)=\hat{\delta} d-E(t)
$$

$$
H(t) \leq \hat{\delta} d+\int_{\Omega} F(u, v) d x \leq\left(\hat{\delta} \frac{r-2}{2}+1\right) \int_{\Omega} F(u, v) d x, \text { para cada } t>0
$$

y

$$
H(t) \geq H(0)=\hat{\delta} d-E(0)>0, H^{\prime}(t)=-E^{\prime}(t) \geq 0, \text { para cada } t>0 .
$$

Lema 3.6. Supongamos que $\left(H_{1}\right)-\left(H_{4}\right)$ y $(3.7)$ se verifican. Entonces existe una constante $C>0$ tal que

$$
\begin{aligned}
|u|_{r}^{q}+|v|_{r}^{q} \leq & C\left[-H(t)-\left|u^{\prime}\right|_{\rho+2}^{\rho+2}-\left|v^{\prime}\right|_{\rho+2}^{\rho+2}+\left\|u^{\prime}\right\|^{2}+\left\|v^{\prime}\right\|^{2}-\|u\|_{1, p}^{p}-\|v\|_{1, p}^{p}\right. \\
& \left.-\|u\|^{2}-\|v\|^{2}-\left(g_{1} \square \nabla u\right)-\left(g_{2} \square \nabla v\right)+|u|_{r}^{r}+|v|_{r}^{r}\right],
\end{aligned}
$$

para $t \geq 0$ y para todo $2 \leq q \leq r$.

Demostración. Usando $\left(H_{2}\right),(3.1),(3.11)$ y $\left(H_{4}\right)$, resulta

$$
\begin{aligned}
\frac{1}{2} l_{1}\|u\|^{2}+\frac{1}{2} l_{2}\|v\|^{2} \leq & E(t)-\frac{1}{\rho+2}\left(\left|u^{\prime}\right|_{\rho+2}^{\rho+2}+\left|v^{\prime}\right|_{\rho+2}^{\rho+2}\right)-\frac{1}{2}\left(\left\|u^{\prime}\right\|^{2}+\left\|v^{\prime}\right\|^{2}\right) \\
& -\frac{1}{p}\left(\|u(t)\|_{1, p}^{p}+\|v(t)\|_{1, p}^{p}\right) \\
& -\frac{1}{2}\left(\left(g_{1} \square \nabla u\right)+\left(g_{2} \square \nabla v\right)\right)+\int_{\Omega} F(u, v) d x \\
= & \hat{\delta} d-H(t)-\frac{1}{\rho+2}\left(\left|u^{\prime}\right|_{\rho+2}^{\rho+2}+\left|v^{\prime}\right|_{\rho+2}^{\rho+2}\right)-\frac{1}{2}\left(\left\|u^{\prime}\right\|^{2}+\left\|v^{\prime}\right\|^{2}\right) \\
& -\frac{1}{p}\left(\|u(t)\|_{1, p}^{p}+\|v(t)\|_{1, p}^{p}\right) \\
& -\frac{1}{2}\left(\left(g_{1} \square \nabla u\right)+\left(g_{2} \square \nabla v\right)\right)+\frac{c_{1}}{r}\left(|u|_{r}^{r}+|v|_{r}^{r}\right) .
\end{aligned}
$$

Utilizando (3.9), se tiene

$$
\begin{aligned}
m\left(\|u\|^{2}+\|v\|^{2}\right) \leq & C_{2}\left[-H(t)-\left(\left|u^{\prime}\right|_{\rho+2}^{\rho+2}+\left|v^{\prime}\right|_{\rho+2}^{\rho+2}\right)-\left(\left\|u^{\prime}\right\|^{2}+\left\|v^{\prime}\right\|^{2}\right)\right. \\
& -\left(\|u(t)\|_{1, p}^{p}+\|v(t)\|_{1, p}^{p}\right) \\
& \left.-\left(\left(g_{1} \square \nabla u\right)+\left(g_{2} \square \nabla v\right)\right)+\left(|u|_{r}^{r}+|v|_{r}^{r}\right)\right],
\end{aligned}
$$

donde $m=\operatorname{mín}\left\{\frac{1}{2} l_{1}, \frac{1}{2} l_{2}\right\}$ y $C_{2}$ es una constante positiva que depende de $\rho, p$ y $c_{1}$. Empleando (3.10) y (3.16), se obtiene (3.14). 
Teorema 3.7 (Explosión de Soluciones). Supongamos que $\left(H_{1}\right)-\left(H_{4}\right)$ se verifican. Si para cada número fijo $\delta<1$, consideremos que $u_{0}, v_{0} \in W_{0}^{1, p}(\Omega), u_{1}, v_{1} \in H_{0}^{1}(\Omega)$ y satisface

$$
E(0)<\delta d \text { y } I(0)<0,
$$

donde d está dado por (3.5). Además supongamos que $\rho<r-2$ y se verifica

$$
\operatorname{máx}\left\{\int_{0}^{\infty} g_{1}(s) d s, \int_{0}^{\infty} g_{2}(s) d s\right\}<\frac{r-2}{r-2+\frac{1}{(1-\hat{\delta})^{2}(r-2)+2 \hat{\delta}(1-\hat{\delta})}},
$$

donde $\hat{\delta}=\operatorname{máx}\{0, \delta\}$. Entonces la solución $(u, v)$ del problema $(1.1)-(1.6)$ explota en tiempo finito, es decir, existe un número positivo finito $T_{*}$ tal que

$$
\lim _{t \rightarrow T_{*}^{-}}\left[\left|u^{\prime}\right|_{\rho+2}^{\rho+2}+\left|v^{\prime}\right|_{\rho+2}^{\rho+2}+\left\|u^{\prime}\right\|^{2}+\left\|v^{\prime}\right\|^{2}+\|u\|^{2}+\|v\|^{2}+\|u\|_{1, p}^{p}+\|v\|_{1, p}^{p}+|u|_{r}^{r}+|v|_{r}^{r}\right]=\infty .
$$

Demostración. Por contradicción, supongamos que la solución del problema (1.1) - (1.6) es global, entonces existe una constante $C>0$ tal que

$$
\left|u^{\prime}\right|_{\rho+2}^{\rho+2}+\left|v^{\prime}\right|_{\rho+2}^{\rho+2}+\left\|u^{\prime}\right\|^{2}+\left\|v^{\prime}\right\|^{2}+\|u\|^{2}+\|v\|^{2}+\|u\|_{1, p}^{p}+\|v\|_{1, p}^{p}+|u|_{r}^{r}+|v|_{r}^{r} \leq C,
$$

para todo $t \geq 0$. Definamos la función

$$
\begin{aligned}
\psi(t)= & H^{1-\sigma}(t)+\frac{\varepsilon}{\rho+1} \int_{\Omega}\left(\left|u^{\prime}\right|^{\rho} u^{\prime} u+\left|v^{\prime}\right|^{\rho} v^{\prime} v\right) d x+\frac{\varepsilon}{2} \int_{\Omega}\left(|\nabla u|^{2}+|\nabla v|^{2}\right) d x \\
& +\varepsilon \int_{\Omega}\left(\nabla u \cdot \nabla u^{\prime}+\nabla v \cdot \nabla v^{\prime}\right) d x
\end{aligned}
$$

donde $0<\varepsilon \ll 1$ que será especificado mas tarde y $0<\sigma<\operatorname{mín}\left\{\frac{1}{2}-\frac{1}{p}, \frac{1}{\rho+2}-\frac{1}{r}\right\}$.

Derivando (3.19), usando $(1.1)-(1.2)$ y $\left(H_{4}\right)$, obtenemos

$$
\begin{aligned}
\psi^{\prime}(t)= & (1-\sigma) H^{-\sigma}(t) H^{\prime}(t) \\
& +\frac{\varepsilon}{\rho+1}\left(\left|u^{\prime}\right|_{\rho+2}^{\rho+2}+\left|v^{\prime}\right|_{\rho+2}^{\rho+2}\right)+\varepsilon \int_{\Omega}\left(\left|u^{\prime}\right|^{\rho} u^{\prime \prime} u+\left|v^{\prime}\right|^{\rho} v^{\prime \prime} v\right) d x \\
& +\varepsilon \int_{\Omega}\left(\nabla u \cdot \nabla u^{\prime}+\nabla v \cdot \nabla v^{\prime}\right) d x+\varepsilon\left(\left\|u^{\prime}\right\|^{2}+\left\|v^{\prime}\right\|^{2}\right) \\
& +\varepsilon \int_{\Omega}\left(\nabla u \cdot \nabla u^{\prime \prime}+\nabla v \cdot \nabla v^{\prime \prime}\right) d x \\
= & (1-\sigma) H^{-\sigma}(t) H^{\prime}(t)+\frac{\varepsilon}{\rho+1}\left(\left|u^{\prime}\right|_{\rho+2}^{\rho+2}+\left|v^{\prime}\right|_{\rho+2}^{\rho+2}\right)+\varepsilon\left(\left\|u^{\prime}\right\|^{2}+\left\|v^{\prime}\right\|^{2}\right) \\
& +\varepsilon r \int_{\Omega} F(u, v) d x-\varepsilon\left(\|u\|^{2}+\|v\|^{2}\right)-\varepsilon\left(\|u\|_{1, p}^{p}+\|v\|_{1, p}^{p}\right) \\
& +\varepsilon\left(\int_{0}^{t} g_{1}(t-s)(\nabla u(s), \nabla u(t)) d s\right. \\
& \left.+\int_{0}^{t} g_{2}(t-s)(\nabla v(s), \nabla v(t)) d s\right) .
\end{aligned}
$$


Desde que $\left(\nabla w_{1}, \nabla w_{2}\right) \geq-\left(\eta\left\|w_{1}\right\|^{2}+\frac{1}{4 \eta}\left\|w_{2}\right\|^{2}\right)$, para $\eta>0$, se obtiene

$$
\begin{aligned}
\int_{0}^{t} g(t-s)(\nabla w(s), \nabla w(t)) d s= & \int_{0}^{t} g(t-s)(\nabla w(s)-\nabla w(t), \nabla w(t)) d s \\
& +\left(\int_{0}^{t} g(s) d s\right)\|w(t)\|^{2} \\
\geq & -\left(\eta(g \square \nabla w)(t)+\frac{1}{4 \eta}\left(\int_{0}^{t} g(s) d s\right)\|w(t)\|^{2}\right) \\
& +\left(\int_{0}^{t} g(s) d s\right)\|w(t)\|^{2} \\
= & \left(1-\frac{1}{4 \eta}\right)\left(\int_{0}^{t} g(s) d s\right)\|w(t)\|^{2}-\eta(g \square \nabla w)(t) .
\end{aligned}
$$

Este resultado en (3.20), implica

$$
\begin{aligned}
\psi^{\prime}(t) \geq & \frac{\varepsilon}{\rho+1}\left(\left|u^{\prime}\right|_{\rho+2}^{\rho+2}+\left|v^{\prime}\right|_{\rho+2}^{\rho+2}\right)+\varepsilon\left(\left\|u^{\prime}\right\|^{2}+\left\|v^{\prime}\right\|^{2}\right)+\varepsilon r \int_{\Omega} F(u, v) d x \\
& -\varepsilon\left(\|u\|_{1, p}^{p}+\|v\|_{1, p}^{p}\right)-\varepsilon\left(G_{1}(t)\|u(t)\|^{2}+G_{2}(t)\|v(t)\|^{2}\right) \\
& -\frac{\varepsilon}{4 \eta}\left[\left(\int_{0}^{t} g_{1}(s) d s\right)\|u(t)\|^{2}+\left(\int_{0}^{t} g_{2}(s) d s\right)\|v(t)\|^{2}\right] \\
& -\varepsilon \eta\left[\left(g_{1} \square \nabla u\right)+\left(g_{2} \square \nabla v\right)\right]
\end{aligned}
$$

para algún número $\eta>0$ a ser determinado más tarde. Por (3.11) y (3.1), resulta

$$
\begin{aligned}
\int_{\Omega} F(u, v) d x= & H(t)-\hat{\delta} d+\frac{1}{\rho+2}\left(\left|u^{\prime}\right|_{\rho+2}^{\rho+2}+\left|v^{\prime}\right|_{\rho+2}^{\rho+2}\right) \\
& +\frac{1}{2}\left(\left\|u^{\prime}\right\|^{2}+\left\|v^{\prime}\right\|^{2}\right)+\frac{1}{p}\left(\|u\|_{1, p}^{p}+\|v\|_{1, p}^{p}\right)+\frac{1}{2} \Gamma(t)
\end{aligned}
$$

y este en (3.21), se obtiene

$$
\begin{aligned}
\psi^{\prime}(t) \geq & \varepsilon\left(\frac{1}{\rho+1}+\frac{r}{\rho+2}\right)\left(\left|u^{\prime}\right|_{\rho+2}^{\rho+2}+\left|v^{\prime}\right|_{\rho+2}^{\rho+2}\right)+\varepsilon\left(1+\frac{r}{2}\right)\left(\left\|u^{\prime}\right\|^{2}+\left\|v^{\prime}\right\|^{2}\right) \\
& +\varepsilon\left(\frac{r-p}{p}\right)\left(\|u\|_{1, p}^{p}+\|v\|_{1, p}^{p}\right)+\varepsilon\left(\mathcal{G}_{1}(t)\|u(t)\|^{2}+\mathcal{G}_{2}(t)\|v(t)\|^{2}\right) \\
& +\varepsilon\left(\frac{r}{2}-\eta\right)\left[\left(g_{1} \square \nabla u\right)+\left(g_{2} \square \nabla v\right)\right]+\varepsilon r H(t)-\varepsilon r \hat{\delta} d,
\end{aligned}
$$

donde $\mathcal{G}_{i}(t)=\frac{r-2}{2}-\left(\frac{r-2}{2}+\frac{1}{4 \eta}\right) \int_{0}^{t} g_{i}(s) d s, i=1,2$. Utilizando la desigualdad (3.9) en la estimativa (3.22), se obtiene

$$
\begin{aligned}
\psi^{\prime}(t) \geq & \varepsilon\left(\frac{1}{\rho+1}+\frac{r}{\rho+2}\right)\left(\left|u^{\prime}\right|_{\rho+2}^{\rho+2}+\left|v^{\prime}\right|_{\rho+2}^{\rho+2}\right)+\varepsilon\left(1+\frac{r}{2}\right)\left(\left\|u^{\prime}\right\|^{2}+\left\|v^{\prime}\right\|^{2}\right) \\
& +\varepsilon\left(\frac{r-p}{p}\right)\left(\|u\|_{1, p}^{p}+\|v\|_{1, p}^{p}\right)+\varepsilon\left(\mathcal{H}_{1}(t)\|u(t)\|^{2}+\mathcal{H}_{2}(t)\|v(t)\|^{2}\right) \\
& +\varepsilon\left(\frac{r-2}{2}(1-\hat{\delta})+(1-\eta)\right)\left[\left(g_{1} \square \nabla u\right)+\left(g_{2} \square \nabla v\right)\right]+\varepsilon r H(t),
\end{aligned}
$$


donde $\mathcal{H}_{i}(t)=\frac{r-2}{2}(1-\hat{\delta})-\left(\frac{r-2}{2}(1-\hat{\delta})+\frac{1}{4 \eta}\right) \int_{0}^{t} g_{i}(s) d s, i=1,2$. Como se verifica $H(t) \geq$ $-E(t)$, se tiene

$$
\begin{aligned}
H(t) \geq & \int_{\Omega} F(u(t), v(t)) d x-\frac{1}{\rho+2}\left(\left|u^{\prime}(t)\right|_{\rho+2}^{\rho+2}+\left|v^{\prime}(t)\right|_{\rho+2}^{\rho+2}\right) \\
& -\frac{1}{p}\left(\|u(t)\|_{1, p}^{p}+\|v(t)\|_{1, p}^{p}\right) \\
& -\frac{1}{2}\left(\left\|u^{\prime}(t)\right\|^{2}+\left\|v^{\prime}(t)\right\|^{2}\right)-\frac{1}{2} \Gamma(u(t), v(t)) .
\end{aligned}
$$

Tomando cualquier números $0<\gamma<1$, desde que $0<(r-2)(1-\hat{\delta})<r$ y $0<(r-p)(1-\hat{\delta})<$ $r$, implica $0<\gamma(r-2)(1-\hat{\delta})<r$ y $0<\gamma(r-p)(1-\hat{\delta})<r$. Por este resultado y utilizando (3.24) en la estimativa (3.23), se deduce

$$
\begin{aligned}
\psi^{\prime}(t) \geq & \varepsilon\left[\frac{1}{\rho+1}+\frac{1}{\rho+2}[r-\gamma(r-2)(1-\hat{\delta})]\right]\left(\left|u^{\prime}\right|_{\rho+2}^{\rho+2}+\left|v^{\prime}\right|_{\rho+2}^{\rho+2}\right) \\
& +\varepsilon\left[1+\frac{1}{2}[r-\gamma(r-2)(1-\hat{\delta})]\right]\left(\left\|u^{\prime}\right\|^{2}+\left\|v^{\prime}\right\|^{2}\right) \\
& +\varepsilon\left(\frac{r-p}{p}-\frac{\gamma(r-p)(1-\hat{\delta})}{p}\right)\left(\|u\|_{1, p}^{p}+\|v\|_{1, p}^{p}\right) \\
& +\varepsilon\left(\mathcal{F}_{1}(t)\|u(t)\|^{2}+\mathcal{F}_{2}(t)\|v(t)\|^{2}\right) \\
& +\varepsilon\left(\frac{r-2}{2}(1-\gamma)(1-\hat{\delta})+(1-\eta)\right)\left[\left(g_{1} \square \nabla u\right)+\left(g_{2} \square \nabla v\right)\right] \\
& +\varepsilon[r-\gamma(r-2)(1-\hat{\delta})] H(t) \\
& +\varepsilon \gamma(r-2)(1-\hat{\delta}) \int_{\Omega} F(u(t), v(t)) d x
\end{aligned}
$$

donde $\mathcal{F}_{i}(t)=\frac{r-2}{2}(1-\gamma)(1-\hat{\delta})-\left(\frac{r-2}{2}(1-\gamma)(1-\hat{\delta})+\frac{1}{4 \eta}\right) \int_{0}^{t} g_{i}(s) d s, i=1,2$. Se debe observar, para obtener (3.25), se ha empleado la propiedad: Si $H \geq A$ y $0 \leq \mu \leq r$, entonces $r H \geq \mu A+(r-\mu) H$.

Si $\delta<0$, entonces $E(0)<0$ y $\hat{\delta}=0$; podemos escoger $\eta=\frac{r-2}{2}(1-\gamma)+1$ y $\gamma \in\langle 0,1\rangle$ tal que $\frac{r-2}{2(1-\gamma)}=\frac{r-2}{2}(1-\gamma)+1$ en $(3.25)$. Si $0 \leq \delta<1$, entonces $E(0)>0$ y $\hat{\delta}=\delta$; podemos escoger $\eta=\frac{(r-2)(1-\delta)^{2}+2 \delta(1-\delta)}{2(1-\gamma)}$ y $\gamma \in\langle 0,1\rangle$ tal que $\frac{r-2}{2}(1-\gamma)(1-\hat{\delta})+1>\frac{(r-2)(1-\delta)^{2}+2 \delta(1-\delta)}{2(1-\gamma)}$ en (3.25). Teniendo en cuenta que $1-l_{i}=\int_{0}^{\infty} g_{i}(s) d s \geq \int_{0}^{t} g_{i}(s) d s, i=1,2$, (3.17) y $\left(H_{4}\right)$, resulta

$$
\begin{aligned}
\psi^{\prime}(t) \geq & \varepsilon \zeta\left[\left|u^{\prime}\right|_{\rho+2}^{\rho+2}+\left|v^{\prime}\right|_{\rho+2}^{\rho+2}+H(t)+\left\|u^{\prime}\right\|^{2}+\left\|v^{\prime}\right\|^{2}+\|u\|^{2}+\|v\|^{2}\right. \\
& \left.+\|u\|_{1, p}^{p}+\|v\|_{1, p}^{p}+\left(g_{1} \square \nabla u\right)+\left(g_{2} \square \nabla v\right)+|u|_{r}^{r}+|v|_{r}^{r}\right], \\
\psi(0)= & H^{1-\sigma}(0)+\frac{\varepsilon}{\rho+1} \int_{\Omega}\left(\left|u_{1}\right|^{\rho} u_{1} u_{0}+\left|v_{1}\right|^{\rho} v_{1} v_{0}\right) d x \\
+ & \frac{\varepsilon}{2} \int_{\Omega}\left(\left|\nabla u_{0}\right|^{2}+\left|\nabla v_{0}\right|^{2}\right) d x+\varepsilon \int_{\Omega}\left(\nabla u_{0} \cdot \nabla u_{1}+\nabla v_{0} \cdot \nabla v_{1}\right) d x>0,
\end{aligned}
$$


donde $\zeta$ es una constante positiva que representa al mínimo de todas las constante positiva de los coeficientes de los términos de la estimativa (3.25) y $\varepsilon>0$ es suficientemente pequeño. En consecuencia

$$
\psi(t) \geq \psi(0)>0, \forall t \geq 0 .
$$

Desde que $\frac{\rho+1}{\rho+2}+\frac{1}{\rho+2}=1$ y $\rho+2<r$, resulta

$$
\begin{aligned}
\left|\int_{\Omega}\left(\left|u^{\prime}\right|^{\rho} u^{\prime} u+\left|v^{\prime}\right|^{\rho} v^{\prime} v\right) d x\right| & \leq\left|u^{\prime}\right|_{\rho+2}^{\rho+1}|u|_{\rho+2}+\left|v^{\prime}\right|_{\rho+2}^{\rho+1}|v|_{\rho+2} \\
& \leq C_{1}\left[\left|u^{\prime}\right|_{\rho+2}^{\rho+1}|u|_{r}+\left|v^{\prime}\right|_{\rho+2}^{\rho+1}|v|_{r}\right] .
\end{aligned}
$$

De donde se tiene

$$
\begin{aligned}
\left|\int_{\Omega}\left(\left|u^{\prime}\right|^{\rho} u^{\prime} u+\left|v^{\prime}\right|^{\rho} v^{\prime} v\right) d x\right|^{\frac{1}{1-\sigma}} & \leq C_{2}\left[\left|u^{\prime}\right|_{\rho+2}^{\frac{\rho+1}{1-\sigma}}|u|_{r}^{\frac{1}{1-\sigma}}+\left|v^{\prime}\right|_{\rho+2}^{\frac{\rho+1}{1-\sigma}}|v|_{r}^{\frac{1}{1-\sigma}}\right] \\
& \leq C_{3}\left[\left|u^{\prime}\right|_{\rho+2}^{\frac{\rho+1}{1-\sigma} \mu}+|u|_{r}^{\frac{\theta}{1-\sigma}}+\left|v^{\prime}\right|_{\rho+2}^{\frac{\rho+1}{1-\sigma} \mu}+|v|_{r}^{\frac{\theta}{1-\sigma}}\right],
\end{aligned}
$$

donde $\frac{1}{\mu}+\frac{1}{\theta}=1$. Escogiendo $\mu=\frac{(1-\sigma)(\rho+2)}{\rho+1}$, implica

$$
\frac{\theta}{1-\sigma}=\frac{\rho+2}{(1-\sigma)(\rho+2)-(\rho+1)}<r .
$$

Utilizando (3.14), obtenemos

$$
\begin{aligned}
\left|\int_{\Omega}\left(\left|u^{\prime}\right|^{\rho} u^{\prime} u+\left|v^{\prime}\right|^{\rho} v^{\prime} v\right) d x\right|^{\frac{1}{1-\sigma}} \leq & C_{4}\left[\left|u^{\prime}\right|_{\rho+2}^{\rho+2}+\left|v^{\prime}\right|_{\rho+2}^{\rho+2}-H(t)+\left\|u^{\prime}\right\|^{2}+\left\|v^{\prime}\right\|^{2}\right. \\
& -\|u\|_{1, p}^{p}-\|v\|_{1, p}^{p}-\|u\|^{2}-\|v\|^{2} \\
& \left.-\left(g_{1} \square \nabla u\right)-\left(g_{2} \square \nabla v\right)+|u|_{r}^{r}+|v|_{r}^{r}\right] .
\end{aligned}
$$

También, se tiene la estimativa

$$
\begin{aligned}
\left|\int_{\Omega}\left(\nabla u \cdot \nabla u^{\prime}+\nabla v \cdot \nabla v^{\prime}\right) d x\right|^{\frac{1}{1-\sigma}} & \leq C_{5}\left[\|u\|^{\frac{1}{1-\sigma}}\left\|u^{\prime}\right\|^{\frac{1}{1-\sigma}}+\|v\|^{\frac{1}{1-\sigma}}\left\|v^{\prime}\right\|^{\frac{1}{1-\sigma}}\right] \\
& \leq C_{6}\left[\|u\|^{\frac{s}{1-\sigma}}+\left\|u^{\prime}\right\|^{\frac{\tau}{1-\sigma}}+\|v\|^{\frac{s}{1-\sigma}}+\left\|v^{\prime}\right\|^{\frac{\tau}{1-\sigma}}\right]
\end{aligned}
$$

donde $\frac{1}{s}+\frac{1}{\tau}=1$. Escogiendo $\tau=2(1-\sigma)$, implica $\frac{s}{1-\sigma}=\frac{2}{1-2 \sigma} \mathrm{y}$

$$
\left|\int_{\Omega}\left(\nabla u \cdot \nabla u^{\prime}+\nabla v \cdot \nabla v^{\prime}\right) d x\right|^{\frac{1}{1-\sigma}} \leq C_{6}\left[\|u\|^{\frac{2}{1-2 \sigma}}+\|v\|^{\frac{2}{1-2 \sigma}}+\left\|u^{\prime}\right\|^{2}+\left\|v^{\prime}\right\|^{2}\right] .
$$

Por consiguiente de (3.27) y(3.28), obtenemos

$$
\begin{aligned}
\psi^{\frac{1}{1-\sigma}}(t)= & {\left[H^{1-\sigma}(t)+\frac{\varepsilon}{\rho+1} \int_{\Omega}\left(\left|u^{\prime}\right|^{\rho} u^{\prime} u+\left|v^{\prime}\right|^{\rho} v^{\prime} v\right) d x\right.} \\
& \left.+\frac{\varepsilon}{2} \int_{\Omega}\left(|\nabla u|^{2}+|\nabla v|^{2}\right) d x+\varepsilon \int_{\Omega}\left(\nabla u \cdot \nabla u^{\prime}+\nabla v \cdot \nabla v^{\prime}\right) d x\right]^{\frac{1}{1-\sigma}} \\
\leq & C_{7}\left[H(t)+\left|u^{\prime}\right|_{\rho+2}^{\rho+2}+\left|v^{\prime}\right|_{\rho+2}^{\rho+2}+\|u\|^{2}+\|v\|^{2}\right. \\
& +\left\|u^{\prime}\right\|^{2}+\left\|v^{\prime}\right\|^{2}+\|u\|^{\frac{2}{1-\sigma}}+\|v\|^{\frac{2}{1-\sigma}} \\
& \left.+\|u\|^{\frac{2}{1-2 \sigma}}+\|v\|^{\frac{2}{1-2 \sigma}}+\|u\|_{1, p}^{p}+\|v\|_{1, p}^{p}+|u|_{r}^{r}+|v|_{r}^{r}\right] .
\end{aligned}
$$


Por (3.13) y(3.18), tenemos

$$
\|u\|^{\frac{2}{1-2 \sigma}},\|v\|^{\frac{2}{1-2 \sigma}} \leq C^{\frac{2}{1-2 \sigma}} \frac{H(t)}{H(0)} \quad \mathrm{y} \quad\|u\|^{\frac{2}{1-\sigma}},\|v\|^{\frac{2}{1-\sigma}} \leq C^{\frac{2}{1-\sigma}} \frac{H(t)}{H(0)} .
$$

Se deduce de (3.29), (3.30), (3.12) y $\left(H_{4}\right)$ que

$$
\begin{aligned}
\psi^{\frac{1}{1-\sigma}}(t) \leq K\left[\left|u^{\prime}\right|_{\rho+2}^{\rho+2}+\left|v^{\prime}\right|_{\rho+2}^{\rho+2}\right. & +\left\|u^{\prime}\right\|^{2}+\left\|v^{\prime}\right\|^{2}+\|u\|^{2}+\|v\|^{2} \\
& \left.+\|u\|_{1, p}^{p}+\|v\|_{1, p}^{p}+|u|_{r}^{r}+|v|_{r}^{r}\right],
\end{aligned}
$$

para todo $t \geq 0$. Combinando (3.26) y (3.31), conseguimos

$$
\psi^{\prime}(t) \geq \frac{\varepsilon \zeta}{K} \psi^{\frac{1}{1-\sigma}}(t), \forall t \geq 0 .
$$

Por integración de (3.32) desde 0 hasta $t$, resulta

$$
\psi^{\frac{\sigma}{1-\sigma}}(t) \geq \frac{1}{\psi^{-\frac{\sigma}{1-\sigma}}(0)-\frac{\varepsilon \zeta \sigma t}{K(1-\sigma)}} .
$$

Esto muestra que $\psi(t)$ explota en tiempo finito

$$
T_{*} \leq \frac{K(1-\sigma)}{\varepsilon \zeta \sigma \psi^{\frac{\sigma}{1-\sigma}}(0)}
$$

y además de (3.31), se obtiene

$$
\lim _{t \rightarrow T_{*}^{-}}\left[\left|u^{\prime}\right|_{\rho+2}^{\rho+2}+\left|v^{\prime}\right|_{\rho+2}^{\rho+2}+\left\|u^{\prime}\right\|^{2}+\left\|v^{\prime}\right\|^{2}+\|u\|^{2}+\|v\|^{2}+\|u\|_{1, p}^{p}+\|v\|_{1, p}^{p}+|u|_{r}^{r}+|v|_{r}^{r}\right]=\infty .
$$

Esto es una contradicción con (3.18). Por tanto, la solución del problema (1.1) - (1.6) explota en tiempo finito.

\section{Conclusión}

En el presente estudio se ha obtenido la no existencia global para ciertas soluciones con energía inicial acotada por cierta constante positiva, el cual generaliza al resultado obtenidos por Quispe et al. [15], y agrega al resultado obtenidos por Hao et al. [6].

\section{Agradecimiento}

Al Consejo Superior de Investigación del Vicerrectorado de Investigación de la UNMSM, por el apoyo financiero otorgado para la ejecución del Proyecto de Estudio de Investigación 2016 con código: 161401081, cuyo resultado es la presente publicación.

\section{Referencias bibliográficas}

[1] Alves, C. O., Cavalcanti, M. M., Domingos Cavalcanti, V. N., Rammaha, M. A., and Toundykov, D. (2009). On existence, uniform decay rates and blow up for solutions of systems of nonlinear wave equations with damping and source terms. Discrete and Continuous Dynamical Systems Series S, 2(3), 583-608. 
[2] Andrade, D., and Mognon, A. (2003). Global Solutions for a System of Klein-Gordon Equations with Memory. Bol. Soc. Paran. Mate., (3s.) v. 21 1/2(2003) : 127-138.

[3] Cavalcanti, M. M., Domingos Cavalcanti, V. N., and Ferreira, J. (2001). Existence and uniform decay for a non-linear viscoelastic equation with strong damping. Mathematical methods in the applied sciences, 24(14), 1043-1053.

[4] Evans, L. C. (2010). Partial differential equations (Vol. 19). American Mathematical Society.

[5] Fabrizio, M., and Morro, A. (1992). Mathematical problems in linear viscoelasticity. Society for Industrial and Applied Mathematics.

[6] Hao, J., Niu, S., and Meng, H. (2014). Global nonexistence of solutions for nonlinear coupled viscoelastic wave equations with damping and source terms. Boundary Value Problems, 2014(1), 250.

[7] Liang, F., and Gao, H. (2011). Exponential energy decay and blow-up of solutions for a system of nonlinear viscoelastic wave equations with strong damping. Boundary Value Problems, 2011(1), 22.

[8] Lions, J. L. (1969). Quelques méthodes de résolution des problemes aux limites non linéaires (Vol. 31). Paris: Dunod.

[9] Liu, W. (2010). General decay and blow-up of solution for a quasilinear viscoelastic problem with nonlinear source. Nonlinear Analysis: Theory, Methods and Applications, 73(6), 18901904 .

[10] Liu, W. (2010). Global existence and uniform decay of solutions for a system of wave equations with dispersive and dissipative terms. Frontiers of Mathematics in China, 5(3), $555-574$.

[11] Liu, W., and Yu, J. (2011). Global existence and uniform decay of solutions for a coupled system of nonlinear viscoelastic wave equations with not necessarily differentiable relaxation functions. Studies in Applied Mathematics, 127(4), 315-344.

[12] Medeiros, L. A., and Miranda, M. M. (1986). Weak solutions for a system of nonlinear Klein-Gordon equations. Annali di Matematica pura ed applicata, 146(1), 173-183.

[13] Miranda, M. M., and Medeiros, L. A. (1987). On the existence of global solutions of a coupled nonlinear Klein-Gordon equations. Funkcialaj Ekvacioj, 30, 147-161.

[14] Nishihara, K., and Kojima, J. (1984). On a global solution of some quasilinear hyperbolic equation. Tokyo journal of mathematics, 7(2), 437-459.

[15] Quispe, T., Santiago, Y., Rojas, S. (2017). No existencia global para un sistema de ecuaciones de onda viscoelásica no lineal. PESQUIMAT 20(1): 19-40 (2017), Revista de la F. C. M. de la Universidad Nacional Mayor de San Marcos.

[16] Renardy, M., Hrusa, W. J., and Nohel, J. A. (1987). Mathematical problems in viscoelasticity, Pitman Monographs and Surveys in Pure and Applied Mathematics, vol. 35.

[17] Segal, I. (1965). Nonlinear partial differential equations in quantum field theory. In Proc. Symp. Appl. Math. AMS (Vol. 17, No. 965, pp. 210-226). 
[18] Sun, F., and Wang, M. (2006). Global and blow-up solutions for a system of nonlinear hyperbolic equations with dissipative terms. Nonlinear Analysis: Theory, Methods and Applications, 64(4), 739-761. 